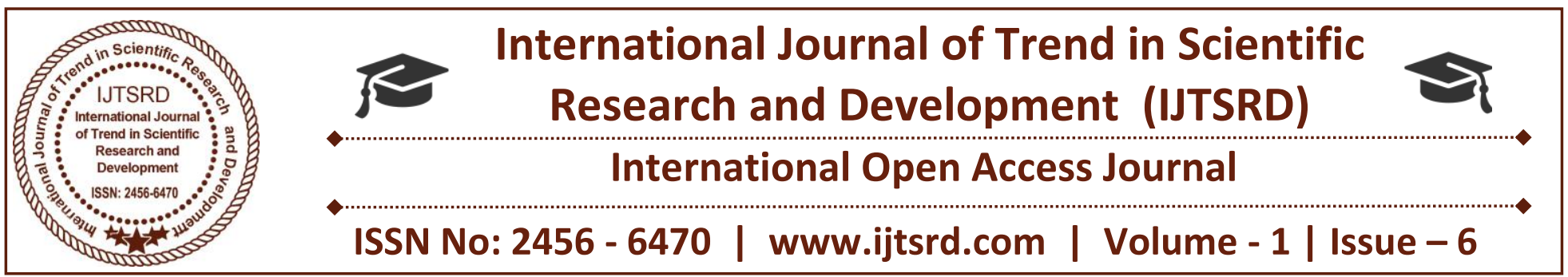

\title{
Video Crime Monitoring
}

\section{Pankaj Sharma}

Department of CSE, Maharishi Arvind

International Institute of Technology, Kota

Parikshit Jhala

Department of HSS, Maharishi Arvind

International Institute of Technology, Kota

\author{
Jagtar Singh \\ Department of MECH., Maharishi Arvind \\ International Institute of Technology, Kota \\ Nirmal Mathur \\ Department of HSS, Maharishi Arvind \\ International Institute of Technology, Kota
}

\begin{abstract}
A CCTV system is not a physical barrier. It does not limit access to certain areas, make an object harder to steal, or a person more difficult to assault and rob. This does not mean it is not an example of situational crime prevention. It is highly situational, and as will be shown, does have some crime prevention capacity in the right situations. Although CCTV has many functions, the primary preventative utility is to trigger a perceptual mechanism in a potential offender. It seeks to change offender perception so the offender believes if he commits a crime, he will be caught. In other words, CCTV aims to increase the perceived risk of capture, a factor which, assuming the offender is behaving in a rational (or limited rational) manner, will de-motivate the potential offender. For this crime prevention process to succeed, So we developed this prototype which shows improved results in terms of crime reporting by the public using mobile devices and improved management of reports due to automation of crime reporting and monitoring.
\end{abstract}

Keywords: CCTV, Crime Reporting, Crime Monitoring

\section{INTRODUCTION}

Nowadays CCTV plays a significant role in protecting the public and implementing security. It is increasingly used by many countries for critical applications, such as bank monitoring, retail control and crime detection, where manual monitoring can be difficult, problematic or unfeasible. As world population increases, traffic control is becoming a critical application for CCTV. CCTV provides a way to monitor multiple cameras internally and analyse generated images to extract useful information about traffic parameters, such as speed, traffic composition, vehicle shapes, vehicle types, vehicle identification numbers and occurrences of traffic violations or road accidents. This paper presents a review on Video crime monitoring by the CCTV technology. It highlights existing architectural and deployment models, as well as the various approaches utilised to analyse generated crime scene.

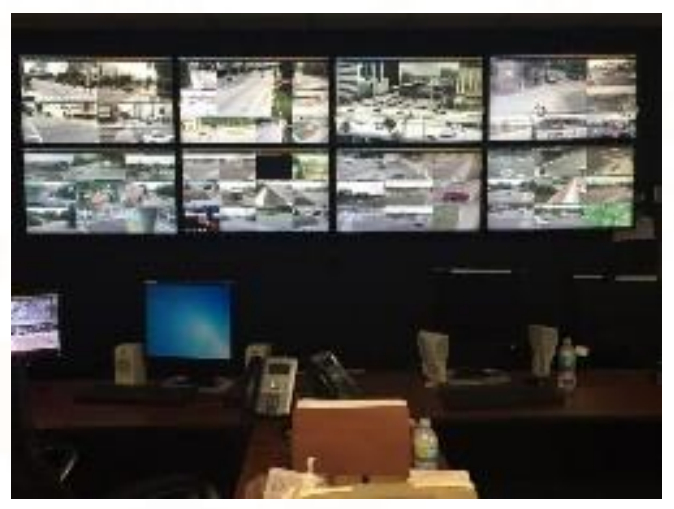

FIG: (1) Video Monitoring 


\section{SYSTEM COMPOSTION}

A CCTV Broadcast is different from regular TV as in regular TV broadcast is meant for people at large however a CCTV broadcast is meant for specific people with specific purposes.

Main components of a CCTV are as follows-

\section{Camera System:}

All CCTV comprises of a group of cameras to monitor and record, videos and Images of specific place. We are using a digital camera as it has high resolution unlimited storage and captured image is automatically chronologically arranged. We have automatic recorders which is equipped with smart detectors which detect any abnormal activity

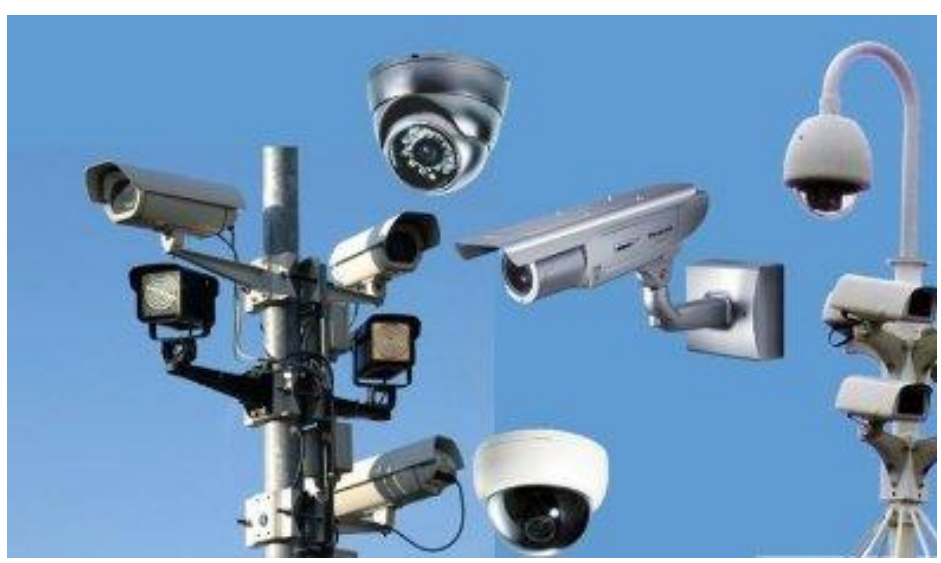

FIG: (2) CCTV cameras

\section{DEPLOYMENT METHOD:}

In order to estimate the traffic load dynamically and control it in real-time. We are using the optimum deployment model. In this model cameras are installed only at particular junction which have been prone to accidents and crimes the positive aspects of this method is that it is as efficient as a site which has cameras installed everywhere. to monitor the entire area, we have pin pointed certain locations which is based on following factors.

We have installed the cameras in urban areas with a vintage point and the broadest pan area which has filed of sight and ample amount of light, also availability of structures. We have also included factors like the geography of the particular area (large building, trees, and curving of the streets)

\section{DATA ANALYSIS APPROACHES:}

We have corroborated both sound based system and image based system.

\section{(A) Sound-based CCTV Systems: -}

A Sound-based CCTV system contains one or more cameras with a directional microphone, a central controller and a video recorder. The sound is recorded constantly accompanied by the video on a memory unit. When the system hears an unusual sound, for instance a "crash-like" sound then, automatically, the pre- and post-accident recorded scenes are sent from the memory to the controller. The controller analyses the situation and identifies possible causes.

\section{(B) Image based CCTV Systems: -}

Image-based CCTV systems have the ability to recognise unusual and abnormal events on roads by analysing digital images and extracting traffic parameters such as speed and traffic composition. Special software tools are usually used to help in recognising vehicle shapes, vehicle types, vehicle identification numbers and occurrence of traffic violations or road accidents.

\section{REACTION APPROACHES:}

We have narrowed down to Automatic Road Traffic Event Monitoring Information System from Manual reaction and Automatic reaction. ARTEMIS the mechanism of such system is that the extended-range camera will notice the vehicles starting to queue and the spot-detection system will confirm this by detecting movement of traffic flow. If it is slower than the pre-defined speed threshold, then the system will send automatic alerts.

\section{CONSIDRATIONS AND LEGAL ISSUES:}

CCTV leads to better traffic control. On the other hand, it is equally important to consider the disadvantages or possible threats that suggest that CCTV should not be used for traffic control. Indeed, there are distinct problems and issues associated with traffic control by CCTV.A big issue associated with CCTV systems is that they are offensive to privacy. Therefore, many countries have introduced a code of conduct for CCTV systems to insure that the systems are operated in a highly professional and private manner. 


\section{CONCLUSION:}

CCTV systems are increasingly used for road monitoring and traffic control systems. A typical CCTV system is composed of a camera system, reviewing displays and a central controller, beside possible additional accessories for lighting and movement controls. It is important to make the right decision regarding the number of cameras to be installed and their location, making sure to avoid possible road obstacles that might affects the quality of captured images

\section{REFERENCES: -}

1) M. McCahill and C. Norris, "CCTV in London," Report Deliverable of Urban Eye Project, 2002.

2) Inter Pacific "All-seeing eye for CCTV surveillance," New Scientist, vol. 208, pp. 23, Nov 13-Nov 19, 2010.

3) N. Buch, M. Cracknell, J. Orwell and S. A. Velastin, "Vehicle localisation and classification in urban CCTV streams," Proc.16th ITS WC, pp. 1-8, 2009.

4) B. C. Welsh and D. P. Farrington, "Effects of Closed-Circuit Television on Crime," The ANNALS of the American Academy of Political and Social Science, vol. 587, pp. 110-135.

5) E. L. Piza, J. M. Caplan and L. W. Kennedy, "Is the Punishment More Certain? An Analysis of CCTV Detections and Enforcement," Justice Q., pp. 